\title{
Nativos digitales y desinformación: un acercamiento desde el sur de Ecuador
}

\section{Digital natives and disinformation: an approach from southern Ecuador}

\author{
Rocío Elizalde-Robles ${ }^{1}$ https://orcid.org/0000-0002-5389-906X, \\ Claudia Rodríguez-Hidalgo 1 https://orcid.org/0000-0003-4004-9389 \\ ${ }^{1}$ Universidad Técnica Particular de Loja, Loja, Ecuador \\ rmelizaldeleutpl.edu.ec, cvrodriguzxdutpl.edu.ec
}

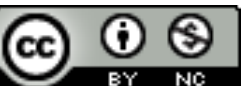

Esta obra está bajo una licencia internacional Creative Commons Atribución-NoComercial 4.0.

$\begin{array}{ll}\text { Enviado: } & 2021 / 06 / 27 \\ \text { Aceptado: } & 2021 / 09 / 28 \\ \text { Publicado: } & 2021 / 11 / 30\end{array}$

\section{Resumen}

Esta investigación indaga en las prácticas de verificación informativa en los nativos digitales, con el uso de herramientas tecnológicas. El objetivo es poner a prueba un chatbot para realizar verificación informativa con elementos de inteligencia artificial; adicionalmente se busca conocer los hábitos de consumo informativo y el conocimiento sobre la desinformación y los sistemas de verificación de contenidos. Se emplea una metodología cualitativa a partir del trabajo con grupo focal. La población se constituye entre personas de sexo masculino y femenino de las generaciones jóvenes, conocidos como nativos digitales, cuyas edades se centran entre la segunda década de vida. Se recolectan datos primarios, a partir de un testeo guiado por tres bloques de preguntas: preguntas de estímulo-inicio, preguntas de desarrollo, bloque de cierre. Los resultados permiten inferir que los nativos digitales gozan de competencia mediática al momento de navegar en entornos digitales, sin que esto implique experticia al momento de interactuar con la desinformación o desarrollar su verificación, para lo cual se reconoce una necesidad formativa. Resulta novedoso y útil, para esta población, el desarrollo y empleo de herramientas que aporten a comprender mejor el fenómeno desinformativo y cómo abordarlo en los entornos cercanos con los cuales se relacionan.

Palabras clave: desinformación, fact-checking, chatbot, verificación informativa, nativos digitales.

Sumario: Introducción, Materiales y Métodos, Metodología, Principales hallazgos, Resultados y Discusión y Conclusiones.

Como citar: Elizalde-Robles, R. \& Rodríguez-Hidalgo, C. (2021). Nativos digitales y desinformación: un acercamiento desde el sur de Ecuador. Revista Tecnológica - Espol, 33(2), 94-108.

http://www.rte.espol.edu.ec/index.php/tecnologica/article/view/834 


\begin{abstract}
This research focuses on information verification practices in digital natives, with the use of technological tools. The objective is to test a chatbot to verify information with elements of artificial intelligence; additionally, it seeks to know the information consumption habits and knowledge about disinformation and content verification systems. A qualitative methodology based on focus group work is used. The population is composed of men and women of young generations, known as digital natives, whose ages are centered between the second decade of life. The primary data were collected from a test guided by three blocks of questions: stimulus start questioning, development questions, closing block. The results allow inferring those digital natives enjoy media skills when navigating digital environments, without implying expertise when interacting with disinformation or developing their verification, for which a training need is recognized. It is novel and useful, for this population, the development and use of tools that contribute to a better understanding of the phenomenon of disinformation and how to address it in the close environments with which they relate.
\end{abstract}

Keywords: disinformation, fact-checking, chatbot, informational verification, digital natives.

\title{
Introducción
}

En las últimas décadas, la evolución de los medios y sistemas digitales han permitido que las comunicaciones en línea se afiancen. Esto obedece, por un lado, a que estos avances de las tecnologías digitales develan un crecimiento en su oferta (Barón et al., 2021); y, por otro, a que a través de estas comunicaciones resultan relaciones basadas en contactos simbólicos, en donde, los dispositivos digitales se constituyen en instrumentos de permanencia (Barrio et al., 2017), especialmente en las generaciones más jóvenes, las cuales han desarrollado distintas habilidades de socialización en estos espacios (Flores \& Browne, 2016).

Las redes sociales, los sistemas de mensajerías, las aplicaciones o los chats dan cuentan de la consolidación de un mundo virtual de comunicaciones de pantallas que transfieren todo tipo de contenidos (López \& Gómez, 2021), lo cual incluye la creciente corriente desinformativa digital (Rodríguez, 2019). De ahí que la discusión sobre la incidencia de la desinformación como un fenómeno inserto en estos medios permanezca vigente (Badillo, 2019) y siga siendo un tema que se analiza desde distintos ámbitos.

Si bien las indagaciones sobre desinformación dan cuenta de su concepción, marco teórico, prácticas, empirismos, modos de producción, distribución en Internet y en las redes sociales desde hace casi una década (Romero, 2013), estos alcanzan un repunte a partir del año 2015, en el contexto de la creciente sobreexposición a climas desinformativos digitales presentes en la esfera mediática (Lotero et al., 2018), en la cual miles de personas hacen frente cada día a contenidos manipulados o inventados con diversos fines. Esto implica la visión de la desinformación como un fenómeno complejo (Innerarity \& Colomina, 2020) que no solo se centra en el uso de la mentira para diversos fines, sino en el poder de expansión que le da Internet, además de visibilizar la dificultad para erradicar las desinformaciones en el mundo digital dado el alcance que logran, una realidad que para ser atendida y entendida requiere conjugar esfuerzos para establecer marcos teóricos multidisciplinarios que permitan profundizar en el abordaje de este fenómeno.

Al presente, encontramos estudios que se proponen comprender mejor la incidencia de la desinformación en las comunicaciones cotidianas. Estos resaltan que se trata de un asunto que requiere de mayor investigación (Pröllonchs, 2021) pues su propagación continúa y logra más alcance por las comunicaciones de interacción digital. 
En las comunicaciones digitales, los espacios de interrelación se basan en redes sociales, las cuales representan para las generaciones más jóvenes, o nativos digitales, un entorno propio (Calderón \& Arteaga, 2020) de relación constante. Se trata de generaciones que crecieron con las tecnologías y medios digitales o conviven directamente con estos ecosistemas (Prensky, 2001), en los cuales encuentran variedad de plataformas, sitios web, comunidades y contenidos con los que pueden interactuar (Mendiguren et al., 2020). Incluso se considera que enfrentan mayores problemas de socialización digital y consumo informativo debido a los desórdenes de información populares en estos ecosistemas, especialmente a lo relacionado con la desinformación (Mendiguren, et al., 2020; Magallón, 2021).

Esta investigación busca conocer la experiencia de los nativos digitales en la interacción con la desinformación. Para ello, se plantea el uso de un chatbot de verificación informativa, como instrumento que busca contraponer los contenidos desinformativos con las prácticas de verificación. A este chatbot se lo denomina Checkingbot y funciona a través de la aplicación WhatsApp. A partir de ello, se pretende identificar las principales problemáticas que genera el contenido desinformativo en este grupo de usuarios, junto a la posibilidad de desarrollar una cultura de verificación, motivando el uso de esta herramienta basada en inteligencia artificial, la cual está al alcance de las comunidades que tienen acceso a los dispositivos móviles.

Checkingbot cuenta con un número de celular y un link web de acceso. En su arquitectura incluye un menú de navegación que permite al usuario acceder a contenidos sobre qué es la desinformación y consultar algún tema de interés o tópico específico, con fines de obtener datos verificados. El chatbot se enmarca en iniciar un canal especializado en verificación y por ello, su personalidad se plantea contrastar la desinformación digital por medio de contenidos provenientes de fuentes verificadas.

\section{Fact-checking para combatir la desinformación \\ Materiales y Métodos}

El fact-checking constituye un elemento que busca aportar en la calidad del trabajo periodístico (Palau, 2017). Se plantea una puesta en práctica de la verificación informativa, por medio de la indagación en datos, fuentes, hechos o discursos que resultan de interés para el debate público (Lotero et al., 2018). Si bien, no es un proceso nuevo para el campo informativo, su auge aumenta en la última década al ser asumido como una estrategia que se plantea frenar a la desinformación, en especial, las denominadas fake news, bulos o falsedades que también han alcanzado mayores rangos de popularidad en los últimos años.

Su puesta en práctica surge en Estados Unidos por medio de impulsos de organismos como FactCheck.org, PolitiFact, FullFact (Nakov et al., 2021), que actualmente agrupan iniciativas de verificación con metodologías que resultan referenciales para quienes se plantean desplegar proyectos fact-checking. En Ecuador las primeras iniciativas de verificación -factchecking- toman fuerza en 2017 en contextos de los discursos políticos-electorales y la pugna presidencial. La presentación de estos medios se da a través de un sitio web y del uso de las redes sociales Facebook y Twitter. Se mantienen activas dos propuestas: Ecuador Chequea (2017) y Ecuador Verifica (2020). Esta última como una coalición de esfuerzos entre entidades educativas y sistemas de información mediáticos.

Las propuestas fact-checking asientan su génesis en observar los asuntos públicos y aquello que manifiestan los principales actores políticos (Rodríguez \& Rangel, 2021). Los procesos de verificación abarcan la selección de frases, partes de un discurso o declaraciones para ser contrastadas con datos (Bekoulis et al., 2021). El interés por la verificación ha llevado al desarrollo de herramientas que resultan relevantes para la identificación de los mensajes 
desinformativos, convirtiendo a la verificación en uno de los grandes retos para los medios digitales (Aguado-Guadalupe \& Bernaola-Serrano, 2020) y sus interacciones.

Su relación con el periodismo se muestra como una oportunidad de mejorar la calidad de los contenidos digitales, aplicando métodos específicos de verificación de datos y discursos. Su empleo ha alcanzado nuevos sitios de trabajo y se impulsa como una manera de hacer frente a los diversos contenidos falsos que generan climas desinformativos en sitios como redes sociales digitales.

Únicamente en 2020, debido a la emergencia sanitaria mundial causada por la Covid19, la desinformación se cataloga como otra pandemia que afecta a comunidades que interactúan por sistemas digitales de comunicación. La Organización Mundial de la Salud emplea el término infodemia para referirse a este tema. La presencia de redes sociales como Facebook, Twitter o Instagram y la creciente búsqueda de información en torno a esta nueva enfermedad, ubica a la desinformación como un símil de una alteración de mayor amplificación, a propósito de uso de las redes sociales (Bacci, 2020), en las comunicaciones digitales.

Según Our World in Data, a inicios de 2021, billones de personas se conectan a plataformas digitales como Facebook, YouTube, WhatsApp, Instagram, WeChat, TikTok, Twitter, entre otras de alta popularidad. Precisamente, estas redes sociales son señaladas por diversos estudios, como los canales de transferencia que hacen que la desinformación logre rangos de exposición antes no registrados. Además, de la propagación de este fenómeno, resultan otras implicaciones, como la formulación de sesgos al momento de abordar un tema o un hecho. El mensaje desinformativo, como un contenido deliberado o interesado, requiere comprobación (Mayora et al., 2017). Al ser consumido por las audiencias digitales, se le otorgan valores de credibilidad incluso a aquellos contenidos no verificados que resultan falsos (García-Marín, 2021) y se encuentran presentes en las comunicaciones digitales de manera activa, conviviendo con otras interacciones realizadas por usuarios cuya presencia en redes digitales también es activa.

Esta situación abre un abanico de complejidades para las audiencias y la comunicación. Las interacciones son, además, con comunidades que responden a las particularidades de universos referidos por los contactos y algoritmos de ecosistemas digitales (González, 2019; García-Marín, 2020), que permiten que cadenas desinformativas tengan presencia, incluso en aplicaciones como WhatsApp (Guallar et al., 2020). En ese contexto, los bots -una herramienta de automatización de contenidos computarizados- son clave a la hora de incrementar el impacto de las campañas de desinformación (Mendiguren et al., 2020). Este aspecto es relevante considerando que actualmente WhatsApp es una de las plataformas sociales más utilizadas a escala mundial (WeAreSocial, 2021).

Los bots se conocen por su naturaleza de automatización y empleo de inteligencia artificial (Túñez et al., 2018). En el marco de estudio de la desinformación, surgen propuestas que buscan emplear los mismos canales por los cuales esta transita, para intentar subvertir los efectos de este fenómeno. Ante la creciente preocupación por el desarrollo de estrategias de confrontación de la incidencia mediática, estas resultan negativas y van de la mano de la desinformación (Golob et al., 2021). Los bots asoman como tecnologías que puede ser utilizadas de forma especializada en la verificación informativa, con metodologías de verificación propias del campo periodístico cuyo empleo pretende aportar en las estrategias para enfrentar el fenómeno desinformativo. 
Lo antes señalado se evidencia en el ejercicio fact-checking para la verificación de hechos, que en los últimos años cobra una importancia trascendental para paliar los efectos nocivos de la desinformación. Entre sus herramientas, el fact-checking se vale de bots cuyos comandos otorgan verificaciones, a partir del ingreso de términos de búsqueda los cuales son averiguados en decenas de bases de datos de medios dedicados a la verificación informativa. Con ello se permite al usuario consultar un tema y recibir recomendaciones para conocer más acerca de la desinformación o directamente verificar sí algún contenido que le cause duda, resulta ser un contenido desinformativo.

\section{Inteligencia Artificial en el fact-checking}

La proliferación indiscriminada de mentiras a través de Internet motiva el desarrollo de herramientas para la verificación informativa. Un proceso que, al desarrollarse de forma tradicional, conlleva mayor tiempo y limitadas acciones en la de búsqueda de información (Vizoso et al., 2018) que permita validar o refutar algún dato, sobre todo los que tienen origen desconocido y se vuelve altamente populares en las conversaciones de redes sociales digitales. En ese sentido, las tecnologías automatizadas, junto a las herramientas de inteligencia artificial constituyen una combinación idónea para realizar la búsqueda y valoración de los contenidos en tiempo real, como un proceso que en cambio, facilita la verificación (Crosas, 2020; López García de Albizu, 2019) de hechos y además, implica un aporte colectivo en la búsqueda de los datos.

En ese contexto surgen los chatbots, los cuales tradicionalmente se emplean con fines comerciales (Janarthanam, 2017), pero que en los últimos años adquieren relevancia como herramientas para confrontar la desinformación, especialmente durante la pandemia por Covid19. Organizaciones periodísticas dedicadas a la verificación informativa incursionan en el uso de chatbots para verificar (Hassan et al., 2017; Salnikova, 2019), tal es el caso de la fundación Maldita.es, que junto a Covid Warriors lanzan un chatbot que interactúa con los usuarios ofreciendo respuestas a dudas con informaciones verificadas, se considera como el primer chatbot de fact-checking de habla hispana (López Linares, 2020).

Otro ejemplo lo impone la International Fact-Checking Network (IFCN) con su proyecto FactChat, que reúne a verificadores de Estados Unidos y medios de comunicación para desmentir las afirmaciones realizadas durante la campaña presidencial de 2020 (IFCN, 2020). Adicional a ello la propagación de fake news sobre la Covid-19 propicia el desarrollo de los chatbots enfocados en desmentir rumores acerca del virus, las medidas de prevención, las posibles curas y temas afines.

Sin embargo la inteligencia artificial también puede ser utilizada para desinformar. Un ejemplo de esto es Grover, sus creadores lo describen como el mejor generador de noticias falsas (Raya, 2019) y que fue utilizado para crear un rumor que luego se hizo masivo sobre un ataque terrorista a la Casa Blanca en el cual el expresidente Obama resultaba herido. La herramienta no ha estado exenta de polémicas, razón por la cual sus creadores afirman que el trasfondo es aprender sobre la generación de fake news y a partir de ello luchar por su erradicación.

Checkingbot es una herramienta cuyo enfoque es hacer frente a la desinformación con la verificación informativa puesta al alcance de los usuarios. En ese contexto, esta investigación da cuenta de los resultados de la experimentación en el uso de tecnologías de la inteligencia artificial en un chatbot y su relacionamiento con los usuarios en la aplicación. Los comandos de Checkingbot emulan indagaciones que permiten resolver las dudas de los usuarios sobre temas que despiertan desconfianza, o demandan de la búsqueda específicas de mayores datos 
para comprender un tema, de manera especial, en aquellos que logran popularidad por sus niveles de viralidad digital. Para ello, se trabaja en la estructura de chatbot que resulte de fácil uso para quienes buscan datos verificados.

La Figura 1 muestra los elementos considerados para el desarrollo de la estructura de un chatbot de verificación informativa. Esta propuesta considera categorías y variables de inteligencia colectiva, desinformación y verificación, por medio de las cuales se crea una distribución y menú de interacción dirigido a usuarios digitales. Desde un almacenamiento, el chatbot aloja información que cumple con los requisitos de ser información validada, la cual se entrega a los usuarios del chatbot, mediante mensajes de respuesta a un menú de consultas. El chatbot se crea para estar disponible en la aplicación de WhatsApp, por ser popular en las comunicaciones del contexto ecuatoriano.

\section{Figura 1}

\section{Estructura del chatbot de verificación informativa «Checkingbot»}

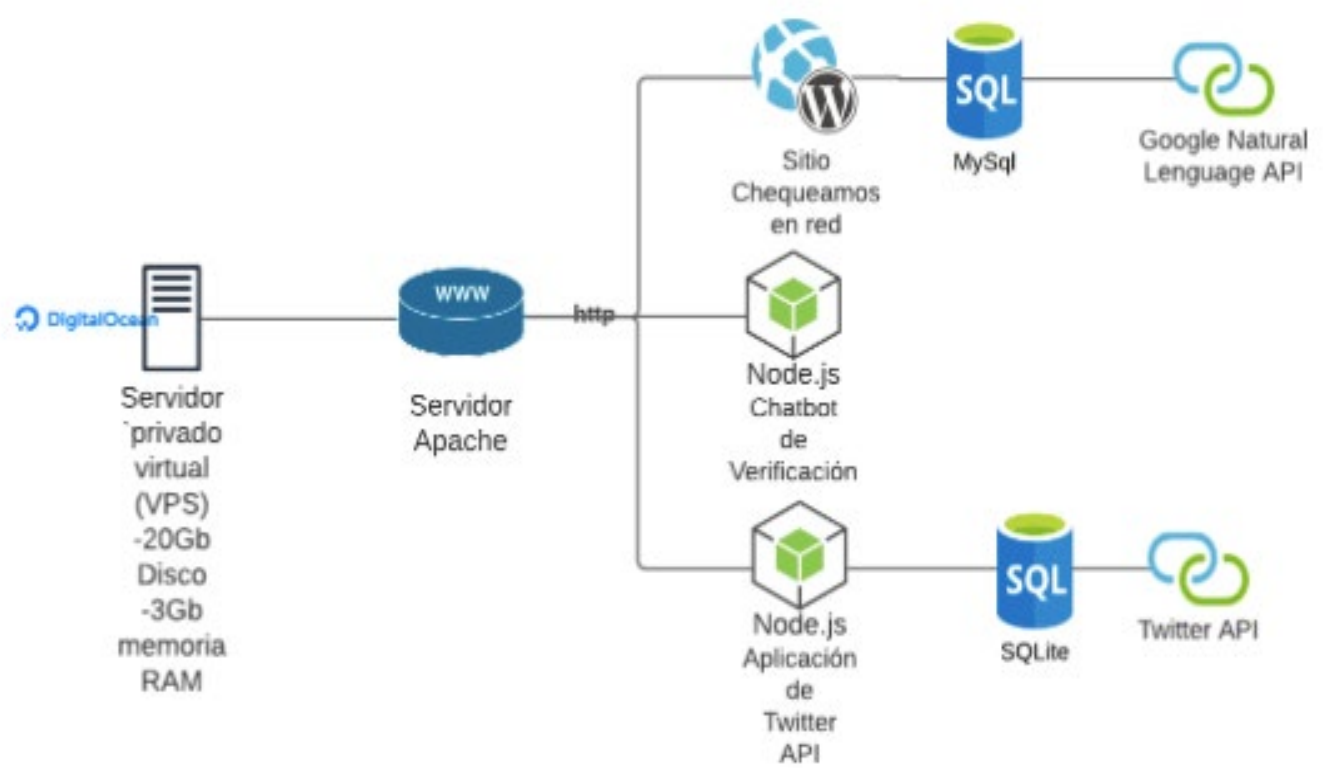

Nota: Se emplearon criterios comunicacionales para la construcción base del chatbot

Estas tecnologías de contrastación de la desinformación son empleadas como estrategias que permiten a los usuarios digitales conseguir datos informativos específicos sobre temas que alcanzan interés colectivo. La Organización Mundial de la Salud, emplea en 2020, un chatbot que provee información vinculada con el Covid-19, mitos sobre esta enfermedad, recomendaciones de bioseguridad, así como desmentidos acerca de las falsedades en torno al desarrollo de las vacunas.

En el caso de Checkingbot su línea editorial incluye temas de coyuntura como: salud, política, economía, elecciones, además, de aquellos que son de interés colectivo vinculados a mitos en salud mental, alimentación, entre otros.

La Figura 2 identifica a las categorías comunes sobre la manera de entender, asociar, asimilar y discutir en las conversaciones incentivadas para abordar a la desinformación por medio de los procesos de verificación como herramientas específicas de contenido. A partir de ello, se identifica que durante el testeo por medio del grupo focal, la interacción con un chatbot de verificación genera experiencias que se sintetizan en cinco categorías comunicativas: 
interfaz, lenguaje, base de datos, contenido verificado, interés de uso. Estos factores resultan clave para motivar su uso en las comunidades digitales que buscan conocer sobre verificación.

\section{Figura 2}

Usabilidad del chatbot en personas jóvenes

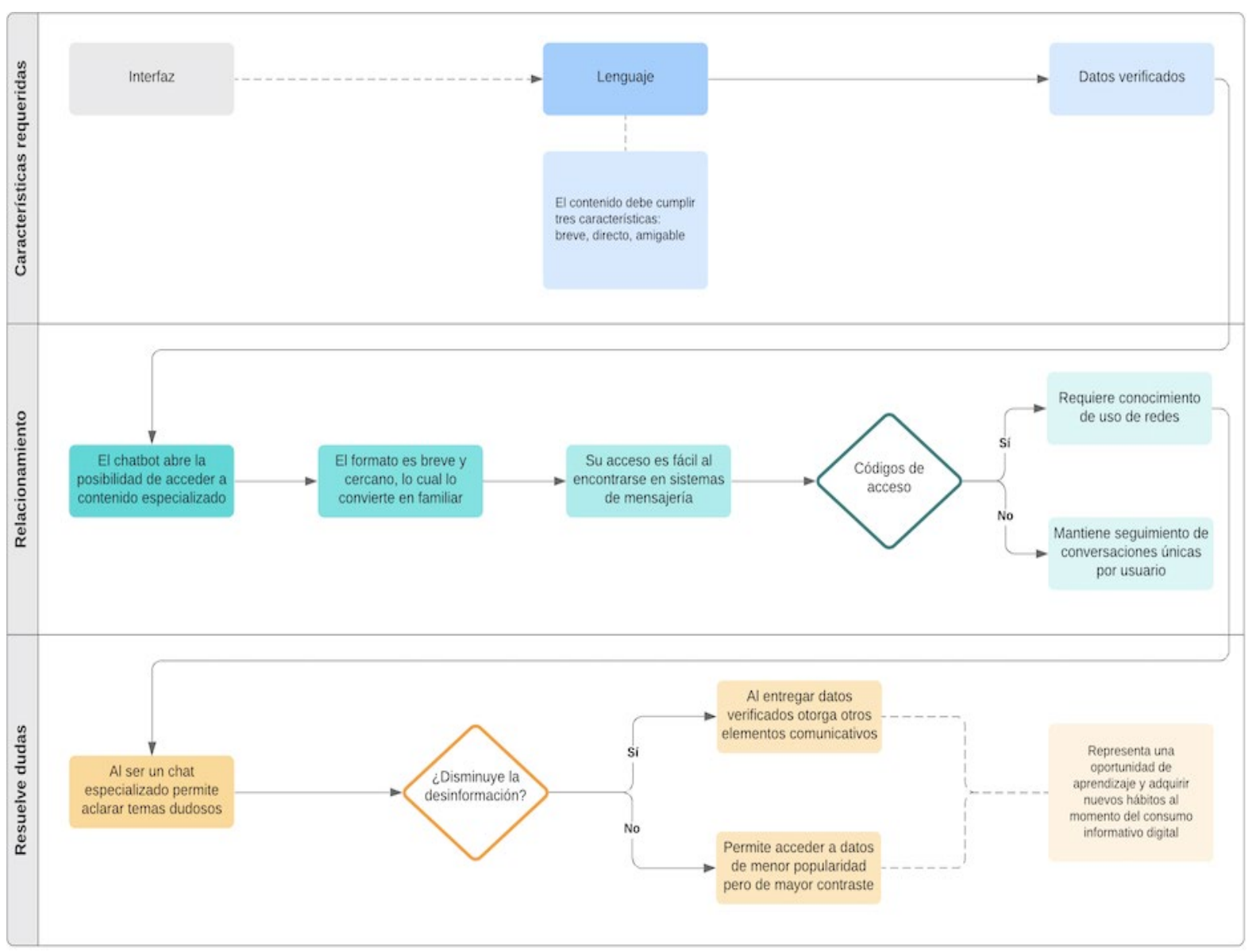

Nota: Los datos se obtuvieron durante un testeo con el chatbot

\section{Metodología}

El objetivo de la investigación es poner a prueba la herramienta tecnológica de factchecking, Checkingbot con un grupo de nativos digitales de la región sur de Ecuador. Para este fin se propuso un grupo focal con un total de 10 nativos digitales, de sexo masculino y femenino, de la zona sur de Ecuador. Se seleccionó esta técnica de levantamiento de información pues posibilita la recopilación de información primaria a partir de las experiencias directas de los participantes del estudio que permitió el acercamiento a la factibilidad del uso de esta herramienta de verificación.

A partir de la construcción de un instrumento de investigación cualitativa se establecieron características de las personas que conformarían la población de estudio, que incluyó: edades, sexo, ubicación geográfica, usuarios que utilicen WhatsApp. Se planteó como objetivo probar y conocer el funcionamiento del software de verificación, Checkingbot, con el fin de indagar en las reacciones, actitudes y experiencias de los usuarios nativos digitales, en torno al uso de aplicaciones de verificación de contenidos digitales de inteligencia colectiva.

Se seleccionó como punto geográfico la provincia de Loja, ubicada al sur de Ecuador, en donde se levantaron los primeros indicadores acerca de la relación entre las poblaciones 
jóvenes, conocidos como como nativos digitales, y su relación con una de las problemáticas comunicacionales actuales: la desinformación digital.

La elección de los perfiles cuidó en conformar una muestra representativa para testear las experiencias e interacciones de los usuarios digitales jóvenes, entre la desinformación, manifestada a través de contenido falso, la verificación informativa y el uso de las tecnologías de verificación como los chatbots. La Tabla 1 detalla las edades de los nativos digitales que participaron en el estudio. Sus ubicaciones son la zona sur de Ecuador. Sus edades también fueron relevantes para el presente estudio, dado los comportamientos manifiestos al momento de interactuar en ecosistemas digitales, por los nativos digitales o poblaciones altamente familiarizadas con las interacciones mediadas en sistemas digitales, como redes sociales y aplicaciones de mensajerías tipo WhatsApp.

Tabla 1

Características de los participantes

\begin{tabular}{|l|c|c|c|}
\hline \multirow{2}{*}{ SEXO } & \multicolumn{3}{c|}{ EDADES } \\
\cline { 2 - 4 } & $19-22$ & $23-26$ & $27-30$ \\
\hline Hombres & 2 & 3 & 1 \\
\hline Mujeres & 2 & 2 & \\
\hline
\end{tabular}

El grupo focal se dividió en tres secciones de trabajo. La primera partió con preguntas de estímulo, se aplicó un banco de 11 cuestionamientos que pretendían conocer las impresiones sobre la existencia de un chatbot como herramienta de verificación de información. A partir del primer cuestionario se abordaron preguntas sobre las experiencias en el uso de redes sociales y aplicaciones es el caso de WhatsApp, como parte de los comportamientos de estos usuarios, en los ecosistemas digitales que usan, además se planteó un sondeo acerca de su conocimiento sobre desinformación, particularmente de fake news. El segundo testeo se basó en aplicar preguntas de desarrollo que buscaron conocer la experiencia sobre el uso e interacción con la herramienta Checkingbot. Para esta parte, la interacción inició con preguntas de tipo intuitivo, hasta conocer los grados de familiaridad con el uso de herramientas de este tipo -chatbots- en WhatsApp. Finalmente, en un tercer momento, se indagó sobre los conocimientos de verificación informativa, interacción con desinformaciones como fake news, su experiencia con estos contenidos, han recibido desinformaciones, si han creído y compartido estos contenidos; adicionalmente, se indagó en sus comportamientos para replicar mensajes e identificar desde qué grupos reciben mensajes desinformativos.

\section{Tabla 2}

Recolección de experiencias en nativos digitales

\begin{tabular}{|l|l|l|}
\hline \multicolumn{3}{|c|}{ ACERCAMIENTO A LA DESINFORMACIÓN EN POBLACIONES JÓVENES } \\
\hline BLOQUE DE PREGUNTAS 1 & BLOQUE DE PREGUNTAS 2 & BLOQUE DE PREGUNTAS 3 \\
\hline Buscan estimular la conversación & Indagan en los conocimientos & Recolectan experiencias sobre la \\
e interacción de manera natural, & sobre tecnologías bot y & relación de los participantes con \\
evitando generar un dominio de & concretamente chatbots de & cesinformaciones, niveles de \\
opinión por algún participante. & verificación, para probar & propagadores, en sus contactos. \\
& Checkingbot. & \multicolumn{2}{|l}{ pontificación de los } \\
\hline
\end{tabular}

A partir de la aplicación de preguntas técnicas descritas en la Tabla 2 se recolectaron los datos, experiencias, inquietudes y reacciones que dan cuenta de cómo llega la desinformación a los nativos digitales y cómo tejen interacciones con tecnologías de chatbots de verificación informativa, a partir de la propuesta de contar con Checkingbot, para averiguar si un contenido corresponde a un hecho noticioso verificado o a una falsedad. 
La participación se realizó de manera activa, voluntaria, neutral y consensuada. Las preguntas de inicio y frases de enganche permitieron una participación motivada, promovida por parte de los asistentes a la investigación. En la recolección de los datos cualitativos para conocer las experiencias de uso del chatbot, se procuró que cada intervención sea natural y se acerque de manera respetuosa, a la realidad con la cual han convivido los usuarios, en los ecosistemas digitales, y el empirismo de la desinformación en mensajes cuyos formatos de difusión corresponde a audios, memes, links o contenidos que resultaron virales y se transfieren por redes sociales o grupos de contacto de los entornos a los cuales los usuarios pertenecen.

\section{Principales hallazgos}

Los nativos digitales manifiestan emplear plataformas digitales para consumir información. Resultan más populares las redes sociales Facebook, Twitter e Instagram. Dos participantes señalan consumir información por medios tradicionales como la televisión y radio de señal local. Al preguntarse si están familiarizados con el término desinformación responden afirmativamente, a partir de la relación con el término fake news, al cual describen como aquellas "noticias" falsas que circulan por los medios sociales digitales y que se enmarcan en temas políticos principalmente.

De la misma forma, reconocen haber recibido en algún momento, durante los últimos 24 meses, algún tipo de contenido falso. La recepción de estos mensajes proviene principalmente de contactos cercanos a sus entornos, como grupos de chat de la familia o algún contacto en su cuenta de Facebook que comparten en sus muros mensajes que resultan falsos. Sin embargo, coinciden en la negativa de compartir este contenido debido a las dudas sobre su veracidad, lo consumen de forma indirecta sin contribuir en la distribución del mismo, en sus contacto o redes sociales, dado que principalmente, el tema no resulta de su interés particular. En ese sentido, señalan a los grupos de chat de amigos o familiares como fuentes poco creíbles a la hora de otorgar valores de veracidad a la información que comparten, cuyas características evidencia viralidad.

\section{Consumo de desinformación}

Los participantes demuestran comportamientos de desafección por el consumo permanente de medios de comunicación tradicionales, así como de medios digitales cuyo tráfico de contenido diario resulta excesivo. Prefieren contenidos breves, cortos, dinámicos y con datos de utilidad.

Respecto a contenidos de dudosa actualidad se observa un conocimiento deficiente tanto en conocer cuáles son los contenidos desinformativos como en conocer cómo verificarlos. Si bien, al momento de buscar información que parece falsa, acuden a cuentas de medios de comunicación para comprobar su veracidad antes de compartir, esta práctica no resulta extendida en todos los participantes, solo en tres de ellos. Los demás mencionan no estar interesados en compartir contenidos entre sus contactos o redes sociales, menos si no están seguros de su veracidad, aunque tampoco se interesan en averiguar si es verdad o no. Este dato de usabilidad de los medios digitales sobre contenido enmarcado en el campo informativo, permite inferir que son usuarios principalmente consumidores con un comportamiento bajo al momento de replicar, por medio de la opción de compartir, aquello que interactúan. La característica principal deriva en un grupo principalmente consumidor de contenido.

En el caso sobre su conocimiento acerca de medios dedicados a la verificación informativa, este grupo indica algunos puntos de referencia a través de medios de verificación como Ecuador Chequea u otros en internet, pero solo en dos de los participantes. De manera 
general, este grupo de nativos desconoce la existencia de organizaciones dedicadas a la verificación de información y más aún proyectos gestados a escala nacional sobre el tema.

\section{Interacción con un chatbot de verificación: Checkingbot}

Durante la socialización de la herramienta Checkingbot se deja a los participantes interactuar de manera intuitiva, sin evidenciar dificultad para el entendimiento de su funcionamiento. En el proceso se recolectaron apuntes de las reacciones y comentarios de los participantes con la personalidad del chatbot. La relación se dio en intervalos de tiempo cortos, minutos, luego de haberse compartido a los participantes el link y número de celular con el cual funciona el chatbot, para que inicien su interacción.

Una vez que lo agregaron a sus celulares, los participantes manifestaron rapidez en comprender cómo se interactúa con un sistema de verificación de contenido en formato de chatbot. En este chatbot de verificación encontraron recomendaciones para conocer qué es desinformación, así como la opción de buscar temas a verificar, por medio de ingresar en el chatbot una frase sobre un hecho que haya sido atendido desde los medios de comunicación, debido a que el chatbot Checkingbot ofrece un menú de navegabilidad que emite instrucciones.

En este proceso se identifica como poco relevante, para los participantes, que el chatbot proporcione links de noticias de diferentes medios, lo cual empata con sus hábitos de consumo de noticias, enmarcados en la preferencia por los contenidos breves. Para ellos, es preferible que este tipo de tecnologías de verificación entre pocos pero buenos enlaces de temas verificados que les permitan conocer de manera rápida sobre qué trata el tema consultado.

Al interactuar con el chatbot Checkingbot, los usuarios lo encuentran funcional. Las respuestas más comunes de las interacciones que se despliegan coinciden en el menú de opciones y se lo enmarca como novedoso y útil. Por ello, indican que podrían mantener la herramienta para consultas específicas entre sus contactos de WhatsApp, aunque no reconocen en este tipo de tecnologías la necesidad de un uso a largo plazo ni de forma permanente, sino para cuestiones muy concretas.

Sin embargo, la propuesta de contar con un chatbot como herramienta para contrarrestar la desinformación les resulta estimulante para la búsqueda de datos específicos. Reconocieron que despierta su interés por el uso de chatbot fuera del ámbito comercial, como ocurre con algunas marcas, y trasladarlo como un canal de comunicación especializado en verificación, que podría convertirse, con el tiempo, en parte de los hábitos de consumo informativo en canales y ecosistemas digitales.

La Figura 3 arroja información consolidada que permite comprender los picos de interacción que resultan comunes en los integrantes de los tres grupos focales, prevalece la experiencia que genera la herramienta con la circulación de contenidos, los temas de coyuntura, los canales de difusión, los nuevos aprendizajes. Esto marca una experiencia de uso de la herramienta de verificación de desinformación mediante inteligencia colectiva, especificada en la propuesta de una chatbot de verificación informativa cuyo funcionamiento se da por medio de WhatsApp.

La experimentación del chatbot permite recolectar la experiencia sobre la interacción con esta herramienta. Este resultado se enmarca en despertar curiosidad en el uso de chatbot de verificación en esta población. Resulta novedosa y factible de utilizar para búsquedas concretas. Algunas observaciones comunes son: las poblaciones jóvenes tienen conocimientos generales sobre la existencia de fake news, sin que alcancen a dimensionar la incidencia de 
estas como atributos del fenómeno más amplio que supone la desinformación, en la comprensión de temas de interés social, su forma de abordar o los sesgos que pueden incidir un contenido desinformativos, acerca de hechos, declaraciones, decisiones públicas o personajes públicos.

\section{Figura 3}

\section{Concordancia en la experiencia de interacción con Checkingbot}

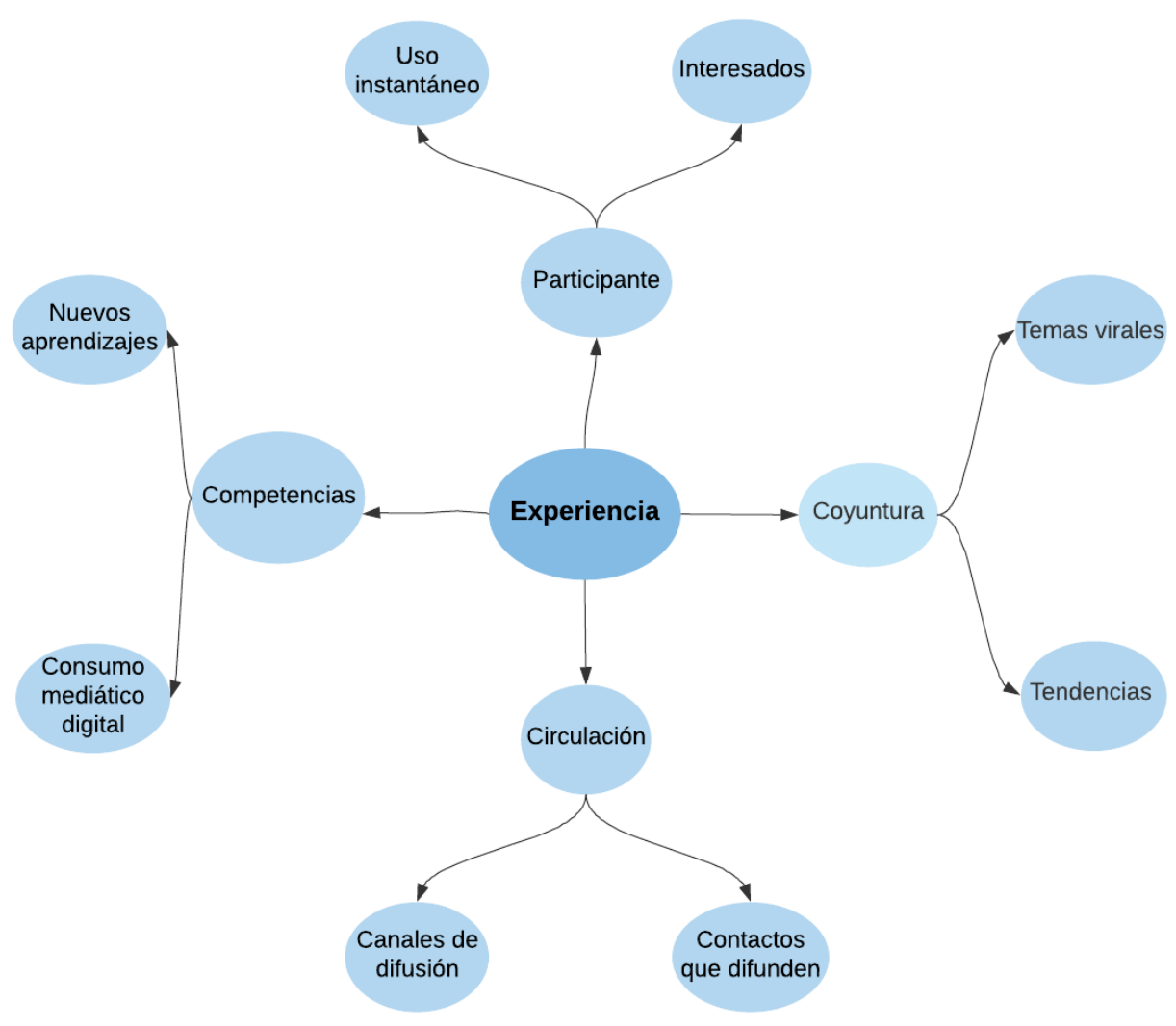

\section{Resultados y Discusión}

Los nativos digitales consideran útil el uso de tecnologías como chatbot de verificación y aunque estas no son conocidas, su uso resulta intuitivo y de fácil acceso por medio de sus celulares y WhatsApp. El uso de un chatbot de verificación informativa permite acceder a una herramienta especializada en contenido verificado. Contar con herramientas de verificación implica en una medida contribuir en desarrollar responsabilidades tanto de medios de comunicación como de los ciudadanos, al momento de consumir medios. Según Palau-Sampio (2017) depende de la percepción de que esta actividad de verificación, sea interpretada como una imparcial, puesto que se convierte en una herramienta que puede renovar la profesión. Esta herramienta pretende ser un canal especializado en verificación, por lo cual, su empleo se puede convertir en momentos específicos de indagación de contenidos.

Para esta población, la desinformación es igual que fake news y su apreciación es que buscan manipular a las personas con contenidos principalmente enfocados en lo político. La Figura 3 lo relaciona a los temas de coyuntura que resultan ser también virales. Al respecto García-Marín (2021), se refiere a que los desórdenes desinformativos constituyen una práctica común de la desinformación, que requiere en la actualidad, fomentar nuevos ejercicios que 
permitan comprender los lenguajes de los contenidos digitales desinformativos. Aunque los nativos digitales muestran habilidades sociales y de interacción con distintos formatos de contenido e incluso herramientas digitales, fortalezas de socialización según Flores \& Brownw, (2016), los nativos digitales principalmente se muestran como consumidores de contenido y no evidenciaron interés en replicar contenidos virales entre sus cuentas o grupos.

Acerca de la verificación informativa, se observa que los participantes de este estudio desconocen la existencia de procesos de verificación fact-checking, por lo que indican que la existencia de estas herramientas es novedosa y a la vez, despierta interés en conocer más sobre su uso como canales especializados en el contenido verificado.

Los nativos digitales advierten como importante que los contenidos que entreguen estas tecnologías deben ser cortos, breves, directos y útiles. Esta apreciación se relaciona con lo expresado en la Figura 1 en la cual se destaca el lenguaje e interfaz empleado para el chatbot. Sobre estos aspectos, autores como Golob et al. (2021) indican que los sistemas mediáticos proporcionan una visión selectiva del mundo, cuya percepción varía incluso por la edad y nivel de educación de las poblaciones, como ocurre en los procesos de contrastación de desinformación digital, verificación informativa y uso de tecnologías chatbot, aún de incipiente creación y aplicación en los campos de verificación.

\section{Conclusiones}

De lo expuesto en este estudio, se infiere entre la relación existente entre los nativos digitales y la desinformación, nociones generales sobre los contenidos falsos sin llegar a dimensionar los alcances de la desinformación como un fenómeno presente en el campo comunicativo. Existe un reconocimiento de fake news en sus comunicaciones digitales sin que ellos contribuyan a expandir su propagación, pero tampoco se preocupen por averiguar sobre su veracidad la interacción es escueta.

Para los nativos digitales el relacionamiento con tecnologías como chatbots de verificación resulta una herramienta de contenido especializado para a ser utilizado en casos específicos. Esta población incluye, en sus hábitos de consumo informativo, una preferencia dominante hacia contenidos ligeros que resulten útiles a sus intereses. Por ello, la herramienta de verificación informativa cumple con esta expectativa al ofrecer datos en un formato corto y dinámico, además de la posibilidad de ser ampliado hacia consultas de interés específico para el usuario a través de los links. Este resultado permite descartar la entrega de una mayoría de links para que aporten en contexto sobre un tema y prevalece el uso de links específicos, breves y útiles.

Contar con la tecnología chatbot de verificación, Checkingbot, abre la posibilidad de ampliar las metodologías fact-checking hacia el desarrollo de otros canales de distribución de contenido verificado, de un tipo de contenido que pretende contrarrestar la desinformación digital con el uso de los mismos canales de interacción directa, como es el caso de WhatsApp. Para ello, se requiere mayores ajustes a los lenguajes que conforman el menú de interacción de estos chatbots hasta lograr que se asemejen lo más posible a contenidos ligeros, breves y útiles, que permitan satisfacer la necesidad de encontrar contenidos veraces en código comunes para los usuarios digitales jóvenes.

La propuesta de verificación por medio de chatbot puede ser mejorada si incluye iniciativas colaborativas en la creación de contenidos, una posibilidad es el periodismo de colaboración que se extienden en iniciativas que conectan proyectos o medios para averiguar 
mayores datos sobre un tema, esto permite alimentar con datos de mayor calidad las entregas que podría hacer Checkinbot.

El chatbot asoma como una herramienta adaptable que pueden potenciarse como una estrategia para contrarrestar la desinformación e impulsar otros hábitos de consumo, más medido y verificado, de temas o hechos que en apariencia, son noticiosos.

Las poblaciones jóvenes muestran conocimientos generales sobre desinformación a partir de los atributos fake news. Estos están asociados principalmente a dos factores: la abundancia de contenidos que circulan en Internet, y de modo particular en las redes sociales. Sin embargo, no se descarta la necesidad formativa sobre estos temas, por lo cual una de las consideraciones de estas herramientas es ser insertada en los sistemas educativos a fin de adiestrar a las poblaciones más jóvenes sobre su uso y a crear una cultura de verificación informativa y difusión responsable de información.

Las limitaciones del estudio fueron en cuanto al alcance de la herramienta chatbot ya que para acceder a esta es necesario ser parte de la población con acceso a Internet y contar con WhatsApp, para así conocer el número de celular o link de acceso al chatbot y poder interactuar. Se evidencia que la herramienta requiere mejorar el lenguaje hasta contar con una comunicación ligera y amigable para el consumo de los nativos digitales. Adicionalmente, dados los comentarios positivos en cuanto la herramienta resulta novedosa como un canal especializado en la verificación informativa, y al ser aún desconocida para poblaciones más amplias, se plantea la consideración de contar con estrategias de difusión, que además explique sobre fact-checking y proyectos de verificación informativa que existen en el país.

Futuras investigaciones podrían enfocarse en otros grupos poblacionales como los adultos mayores, con el fin de desarrollar una visión más amplia acerca del uso de la inteligencia artificial en la verificación informativa, así como trabajar en desarrollar una personalidad más específica del chatbot o mejorar la base de datos que utiliza para mostrar contenidos verificados.

\section{Reconocimientos}

Este trabajo ha sido financiado parcialmente por el Proyecto de Investigación Detección de fake news a través de métodos de inteligencia colectiva, del grupo de Investigación Comunicación, Poder y Ciudadanía en Red, de la Universidad Técnica Particular de Loja.

Los autores desean expresar su agradecimiento a Anthony Cabrera, por su participación en el trabajo en el desarrollo de la herramienta de chatbot de verificación informativa, CheckingBot.

\section{Referencias}

Aguado-Guadalupe, G., Bernaola-Serrano, I. (2020). Verificación en la infodemia de la Covid-19. El caso Newtral. Revista Latina de Comunicación Social. 78, 289-308. https://www.doi.org/10.4185/RLCS2020-1478.

Bacci, S. (2020). COVID-19. Una pandemia de desinformación. Med Interna. 31-34. Medicina Interna, Educación médica y comunidad. https://svmi.web.ve/ojs/index.php/medint/article/view/539/527.

Bekoulis, G., Papagiannopoulou, C., Deligiannis, N., (2021). Undertanding the Impact of Evidence-Aware Sentence Selection for Fact-checkign. Workshop on NLP for Internet Freedom, 23-28. Association for Computational Linguistics. https://www.aclweb.org/anthology/2021.nlp4if-1.4.pdf. 
Badillo A. (2019). La sociedad de la desinformación: propaganda, fake news y la nueva geopolítica de la información. Documento de trabajo. Real Instituto Elcano. https:/www.almendron.com/tribuna/wpcontent/uploads/2019/05/la-sociedad-de-la-desinformacion.pdf.

Barón, M., Duque, A., Mendoza, F., Quintero, W. (2021). Redes sociales y relaciones digitales, una comunicación que supera el cara a cara. Revista Internacional de Pedagogía e Innovación Educativa. https://editic.net/ripie/index.php/ripie/article/view/29/24.

Barrio, M., Borges, E., García, F. (2017). Redes sociales y jóvenes en la era algorítmica. Vidas registradas. TELOS dossier. https://dspace.stir.ac.uk/handle/1893/25849\#.YMuwzS2xB8c.

Calderón, J., R., Arteaga (2020). Nativos digitales: perspectivas de su rol como consumidor. Journal Business Science. 15-22. https://revistas.uleam.edu.ec/index.php/business_science/article/view/47/45.

Crosas, M. (2020). Los chatbots que combaten las “fake news” sobre el coronavirus. El Nacional.Cat. https://www.elnacional.cat/es/salud/chatbots-fake-news-coronavirus_494616_102.html.

Flores, P., Browne, R. (2016). Jóvenes y patriarcado en la sociedad TIC: Una reflexión desde la violencia simbólica de género en redes sociales. Revista Latinoamericana. DOI:10.11600/1692715x.1510804082016.

García-Marín, D. (2021). Las fake news y los periodistas de la generación z. Soluciones post-millennial contra la desinformación. Vivat Academia. Revista de Comunnicación, 154. 37-63. http://doi.org/10.15178/va.2021.154.e1324.

García-Marín, D. (2020). Infodemia global. Desórdenes informativos, narrativas fake y fact-checking en la crisis de la Covid-19. Profesional de la Información. Volumen 20. Nro. 4. https://doi.org/10.3145/epi.2020.jul.11.

González, M. (2019). Fake News: desinformación en la era de la sociedad de la información. Ámbitos. Revistas Internacional de Comunicación. DOI: http://dx.doi.org/10.12795/Ambitos.2019.i45.03.

Golob, T., Makarovič, M., \& Rek, M. (2021). Meta-reflexicibidad para la resiliencia contra la desinformación. Comunicar, 66, 107-118. https://doi.org/10.3916/C66-2021-09.

Guallar, J., Codina, L., Freixa, P., Pérez-Montoro, M. (2020). Desinformación, bulos, curación y verificación. Revisión de estudios en Iberoamérica 2017-2000. Telos: revista de Estudios Interdisciplinarios en Ciencias Sociales, 22. Venezuela. DOI: www.doi.org/10.36390/telos223.09.

Hassan, N., Adair, B., Hamilton, J. T., Li, C., Tremayne, M., Yang, J., \& Yu, C. (2017). The Quest to Automate Fact-Checking. KDD '17: Proceedings of the 23rd ACM SIGKDD International Conference on Knowledge Discovery and Data Mining, 1803. https://doi.org/https://doi.org/10.1145/3097983.3098131.

Innerarity, D. Colomina, C. (2020). Introducción: desinformación y poder, la crisis de los intermediarios. Revista CIDOB Dafers Internacionals. DOI: doi.org/10.24241/rcai.2020.124.1.7.

IFCN. (2020). FactChat: El poder está en tus manos - Poynter. https://www.poynter.org/ifcn/factchates/

Janarthanam, S. (2017). Hands of chatbots an conversacional UI development. Packt Publishing Ltd.

López, N., y Gómez L. (2021). Tendencias de cambio en el comportamiento juvenil ante los media: Millennials vs Generación Z. Estudios sobre el Mensaje Periodístico. Ediciones Complutense. https://dx.doi.org/10.5209/esmp.70170.

Lotero-Echeverri, G. Romero-Rodríguez, L. Pérez-Rodríguez, A. (2018). 'Fact-Checking'vs 'Fake News': Periodismo de confirmación como recurso de la competencia mediática contra la desinformación. Index comunicación. Nro. 8.

López Linares, C. (2020, 18 diciembre). Con lenguaje coloquial, formatos atractivos e inteligencia. Fundación Gabo. https://fundaciongabo.org/es/blog/laboratorios-periodismo-innovador/con-lenguaje-coloquial- 
formatos-atractivos-e-inteligencia.

López, X. Vizoso., Á., Pérez, S. (2019). Iniciativas de verificación en el escenario de la desinformación. Journal. Volumen 15. Nro. 3. DOI: 10.25200/BJR.v15n3.2019.1215.

Magallón, R. (2021). Desinformación y pandemia. La nueva realidad. Comunicación y Hombre. Número 17. DOI: https://doi.org/10.32466/eufv-cyh.2021.17.664.385-387.

Mayora, J., Parratt, S., Morata, M. (2017). Desinformación, manipulación y credibilidad periodística: una perspectiva histórica. Historia y comunicación social. 395-409. https://dx.doi.org/10.5209/hics.66267.

Mendiguren, T., Pérez, J., Meso, K. (2020). Actitud ante las Fake News: Estudio del caso de los estudiantes de la Universidad del País Vasco. Revista de Comunicación, Volumen 19. Nro. 1. DOI: https://doi.org/10.26441/RC19.1-2020-A10.

Nakov, P., Corney, D., Hasanain, M., Alam, F., Elsayed, T., Barrón, A., Papatti, P., Shaar, S., Da San Martino, G., (2021). Automated Fact-Checking for Assisting Human Fact-Checkers. https://arxiv.org/pdf/2103.07769.pdf.

Our World in Data (2021). Number of people using social media platforms, 2017 at 2019. https://ourworldindata.org.

Organización Mundial de la Salud. (2020). Guía de la infodemia sobre la covid 19: promover comportamientos saludables y mitigar los daños derivados de la información incorrecta y falsa. https://www.who.int/es/news/item/23-09-2020-managing-the-covid-19-infodemic-promoting-healthybehaviours-and-mitigating-the-harm-from-misinformation-and-disinformation.

Palau-Sampio, D., (2017). Fact-checking y vigilancia del poder: La verificación del discurso público en los nuevos medios de América Latina. Communication \& Society, 347-365. Doi: 10.15581/003.31.3.347365.

Prensky, M. (2001). Digital Natives, Digital Immigrants Part 2: Do They Really Think Differently? On the Horizon, 9(6), 1-6. https://doi.org/10.1108/10748120110424843.

Pröllochs, N. (2021). Community-Based Fact-Cheching on Twitter's Birdwatch Platform. University of Giessen, Germany.

Raya, A. (2019). Una nueva IA es capaz de crear fake news muy fáciles de creer. El Español. https://www.elespanol.com/omicrono/software/20190531/nueva-ia-capaz-crear-news-facilescreer/402710951_0.html.

Rodríguez, C. (2019). No digas fake news, di desinformación: una revisión sobre el fenómeno de las noticias falsas y sus implicaciones. Comunicación Nro. 40. DOI: http://dx.doi.org/10.18566/comunica.n40.a05.

Romero, L. (2013). Hacia un estado de la cuestión de las investigaciones sobre desinformación/misinformación. Correspondencias \& Análisis. Nro.3. DOI: https://doi.org/10.24265/cian.2013.n3.14.

Rodríguez, M., Rangel, P., (2021). El fact-checking como práctica de verificación del discurso público en América Latina y Venezuela. Un estado de la cuestión. Revista Invecom. Estudios transdisciplinarios en comunicación y sociedad. http://revista.invecom.org/index.php/ojs/article/view/46/147.

Salnikova, L. (2019). Robots Versus Journalists: Does Journalism Have a Future? Theoretical and Practical Issues of Journalism, 8(4), 668-678. https://doi.org/10.17150/2308-6203.2019.8(4).668-678.

Túñez, J., Toural, C., Cacheiro, S., (2018). Uso de bots y algoritmos para automatizar la redacción de noticias: percepción y actitudes de los periodistas en España. El profesional de la información. https://revista.profesionaldelainformacion.com/index.php/EPI/article/view/epi.2018.jul.04/40574.

WeAreSocial. (2021). Digital 2021. https://wearesocial.com/digital-2021 